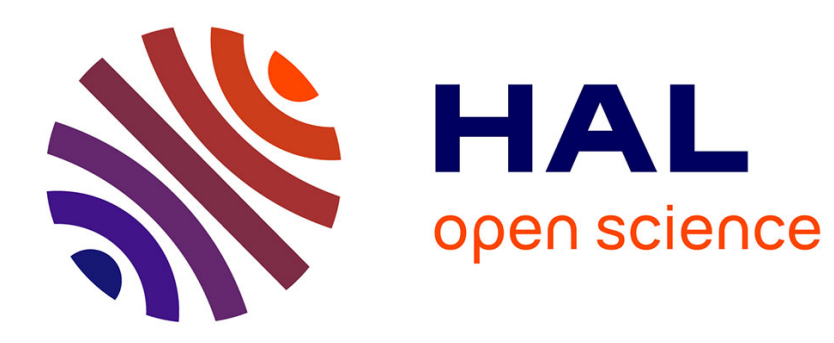

\title{
Analytical nuclear gradients for the MP2-R12 method
} Wim Klopper, Elena Kordel, Cristian Villani

\section{To cite this version:}

Wim Klopper, Elena Kordel, Cristian Villani. Analytical nuclear gradients for the MP2-R12 method. Molecular Physics, 2008, 105 (19-22), pp.2565-2576. 10.1080/00268970701594013 . hal-00513132

\section{HAL Id: hal-00513132 \\ https://hal.science/hal-00513132}

Submitted on 1 Sep 2010

HAL is a multi-disciplinary open access archive for the deposit and dissemination of scientific research documents, whether they are published or not. The documents may come from teaching and research institutions in France or abroad, or from public or private research centers.
L'archive ouverte pluridisciplinaire HAL, est destinée au dépôt et à la diffusion de documents scientifiques de niveau recherche, publiés ou non, émanant des établissements d'enseignement et de recherche français ou étrangers, des laboratoires publics ou privés. 


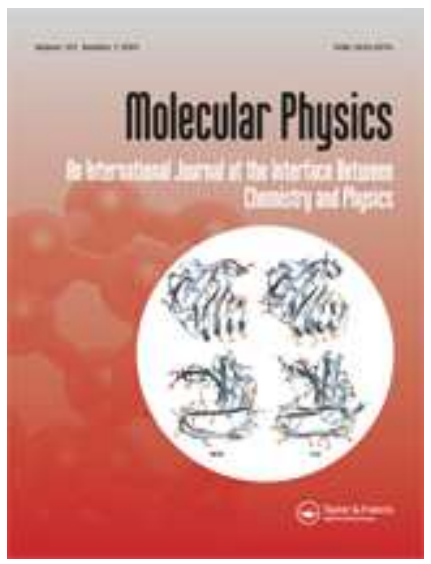

\section{Analytical nuclear gradients for the MP2-R12 method}

\begin{tabular}{|c|c|}
\hline Journal: & Molecular Physics \\
\hline Manuscript ID: & TMPH-2007-0145.R1 \\
\hline Manuscript Type: & Full Paper \\
\hline $\begin{array}{l}\text { Date Submitted by the } \\
\text { Author: }\end{array}$ & 17-Jul-2007 \\
\hline Complete List of Authors: & $\begin{array}{l}\text { Klopper, Wim; University of Karlsruhe } \\
\text { Kordel, Elena; University of Karlsruhe } \\
\text { Villani, Cristian; University of Karlsruhe }\end{array}$ \\
\hline Keywords: & Nuclear gradient, analytical derivatives, MP2-R12 theory \\
\hline \multicolumn{2}{|c|}{$\begin{array}{l}\text { Note: The following files were submitted by the author for peer review, but cannot be converted } \\
\text { to PDF. You must view these files (e.g. movies) online. }\end{array}$} \\
\hline TMPH-2007-0145.tex & \\
\hline
\end{tabular}

\section{scholarONE" \\ Manuscript Central}




\title{
Analytical nuclear gradients for the MP2-R12 method
}

\author{
E. Kordel, C. Villani and W. Klopper \\ Lehrstuhl für Theoretische Chemie, Institut für Physikalische Chemie, \\ Universität Karlsruhe (TH), D-76128 Karlsruhe, Germany
}

(Dated: July 17, 2007)

\begin{abstract}
Analytical energy gradients have been implemented in the DALTON program at the level of explicitly correlated second-order Møller-Plesset perturbation theory (MP2-R12). The implementation has been accomplished for standard approximation A using an auxiliary basis which is the same as the orbital basis for the resolution-of-the-identity, with and without frozen core. As a test calculation, the geometries of nine stationary points on the potential energy surface of the water dimer have been optimized and the results are compared with earlier studies.
\end{abstract}




\section{INTRODUCTION}

One of the major hurdles for accurate electronic structure calculations is the slow $(l+1)^{-3}$ convergence to the basis set limit of conventional orbital based correlation methods. This can be explained with the inadequate description of the correlation cusp (or rather the Coulomb hole) provided by these methods. By including the interelectronic distance in the wave function one obtains a correct description of the latter at and around the correlation cusp. These so called explicitly correlated methods were introduced by Hylleraas ${ }^{1}$ in 1929 for the He atom and have been made applicable to a broader spectrum of chemical systems by the introduction of the so called MP2-R12 methods (explicitly correlated second-order MøllerPlesset perturbation theory with terms linear in the interelectronic distance) in $1987 .{ }^{2}$ Since then there has been much progress in this field. New Ansätze and more accurate integral approximations have been developed ${ }^{3-6}$ and new correlation factors have been introduced,,$^{7,8}$ which allow together with more efficient implementations ${ }^{9,10}$ for more accurate and faster calculations.

With a few exceptions ${ }^{11}$ all of these works have been concerned with a better description of the ground state energy at a fixed nuclear configuration. Having introduced the analytical calculation of first-order molecular properties in reference 12, we will now present formulas and first results for the analytical calculation of nuclear gradients for the MP2-R12/A method. In the next section we will begin by presenting the MP2-R12 Hylleraas functional. We will then introduce the Lagrange functional that will serve as a starting point for deriving the different parts of the molecular gradient, namely the one-electron term, the reorthonormalization term and the two-electron term. The first one has been discussed in detail in reference 12, the two latter will be discussed in the course of this work. As test calculations we will present the results of geometry optimizations of nine different stationary points on the potential energy surface of the water dimer and compare them with several previous studies. ${ }^{13-15}$ 


\section{COMPUTATIONAL METHODS}

\section{A. The MP2-R12 Hylleraas functional}

Throughout this paper $i, j, \ldots$ denote active occupied, $I, J, \ldots$ inactive occupied, $a, b \ldots$ virtual and $p, q, \ldots$ general orbitals. Furthermore we assume that all vectors and matrices are real. Since we restrict our considerations to cases in which orbital and auxiliary basis are the same, no more indices are needed. The MP2-R12 energy can be written as the sum of two independent contributions, the first being the standard MP2 contribution, the second being the R12 contribution

$$
E_{\mathrm{MP} 2-\mathrm{R} 12}=E_{\mathrm{MP} 2}+E_{\mathrm{R} 12}
$$

The R12 contribution to the MP2-R12 energy is obtained from requiring the MP2-R12 Hylleraas functional

$$
F_{\mathrm{R} 12}=\sum_{i j}\left\{\tilde{\mathbf{R}}^{i j} \cdot \mathbf{B}^{i j} \mathbf{R}^{i j}+2 \mathbf{V}^{i j} \cdot \tilde{\mathbf{R}}^{i j}\right\}
$$

to be stationary with respect to the $\mathrm{R} 12$ amplitudes collected in the vector $\mathbf{R}^{i j}$ with the symmetry property

$$
\left[\mathbf{R}^{i j}\right]_{k l}=\left[\mathbf{R}^{j i}\right]_{l k} .
$$

We have introduced the slightly modified R12 amplitudes $\left[\tilde{\mathbf{R}}^{i j}\right]_{k l}$, which are given by

$$
\left[\tilde{\mathbf{R}}^{i j}\right]_{k l}=\left[2 \mathbf{R}^{i j}-\mathbf{R}^{j i}\right]_{k l} .
$$

The matrix $\mathbf{B}^{i j}$ and the vector $\mathbf{V}^{i j}$ can be expressed by

$$
\left[\mathbf{B}^{i j}\right]_{k l, m n}=\left\langle k l\left|r_{12} \hat{Q}_{12}\left(\hat{f}_{12}-\epsilon_{i}-\epsilon_{j}\right) \hat{Q}_{12} r_{12}\right| m n\right\rangle
$$

and

$$
\left[\mathbf{V}^{i j}\right]_{k l}=\left\langle k l\left|r_{12} \hat{Q}_{12} r_{12}^{-1}\right| i j\right\rangle,
$$

respectively. $\hat{f}_{12}=\hat{f}_{1}+\hat{f}_{2}$ is the sum of the Fock operators for electrons 1 and 2 and $\epsilon_{i}$ are the orbital energies. Throughout the paper, only Ansatz 1 will be used. In this case the projection operator reads

$$
\hat{Q}_{12}=\left(1-\hat{P}_{1}\right)\left(1-\hat{P}_{2}\right)
$$

with $\hat{P}=\sum_{p}|p\rangle\langle p|$ the projector on the whole set of MOs.

Minimizing the Hylleraas functional with respect to the R12 amplitudes yields the MP2-R12 energy 


$$
E_{\mathrm{MP} 2-\mathrm{R} 12}=E_{\mathrm{MP} 2}+\sum_{i j} \mathbf{V}^{i j} \cdot \tilde{\mathbf{R}}^{i j}
$$

For later use we define the density matrices

$$
\mathbf{D}=\sum_{i j} \mathbf{D}^{i j} \quad\left[\mathbf{D}^{i j}\right]_{k l, m n}=\left[\mathbf{R}^{i j}\left(\tilde{\mathbf{R}}^{i j}\right)^{T}\right]_{k l, m n}
$$

and write the R12 contribution to the MP2-R12 Hylleraas functional as

$$
F_{\mathrm{R} 12}=\sum_{i j}\left\{\operatorname{tr}\left[\mathbf{B}^{i j} \mathbf{D}^{i j}\right]+2 \mathbf{V}^{i j} \cdot \tilde{\mathbf{R}}^{i j}\right\}
$$

We restrict our considerations to approximation $\mathrm{A}$, in which the matrix $\mathbf{B}^{i j}$ is given by

$$
\left[\mathbf{B}^{i j}(A)\right]_{k l, m n}=\frac{1}{2}\left\langle k l\left|r_{12} \hat{Q}_{12}\left[\hat{T}_{12}, r_{12}\right]+\left[r_{12}, \hat{T}_{12}\right] \hat{Q}_{12} r_{12}\right| m n\right\rangle
$$

Here $\hat{T}_{12}=\hat{T}_{1}+\hat{T}_{2}$ denotes the sum of the kinetic energy operators of electron 1 and 2 . Using the standard approximation ${ }^{16}$ we obtain

$$
\begin{aligned}
{\left[\mathbf{V}^{i j}\right]_{k l} } & =\delta_{k}^{i} \delta_{l}^{j}-\sum_{p q} r_{k l}^{p q} g_{p q}^{i j} \\
{\left[\mathbf{B}^{i j}\right]_{k l, m n} } & =\delta_{k}^{i} \delta_{l}^{j}-\frac{1}{2} \sum_{p q} r_{k l}^{p q} t_{p q}^{m n}-\frac{1}{2} \sum_{p q} r_{m n}^{p q} t_{p q}^{k l}
\end{aligned}
$$

with the two-electron integrals

$$
\begin{aligned}
r_{p q}^{r s} & =\left\langle p q\left|r_{12}\right| r s\right\rangle, \\
g_{p q}^{r s} & =\left\langle p q\left|r_{12}^{-1}\right| r s\right\rangle, \\
t_{p q}^{r s} & =\left\langle p q\left|\left[\hat{T}_{12}, r_{12}\right]\right| r s\right\rangle .
\end{aligned}
$$

\section{B. The MP2-R12 Lagrangian}

In order to exploit Wigner's $2 n+1$ and $2 n+2$ rules, we use the Lagrangian as a starting point for deriving the equations for the R12 contribution to the MP2-R12 gradient following the derivation and notation of reference 17. The R12 Hylleraas functional is stationary with respect to the R12 amplitudes but not with respect to the MO coefficients $\mathbf{C}$. Thus, we require that the Brillouin condition $\left(f_{a i}=0, f_{a I}=0\right)$ is satisfied and that the core-valence 
Fock matrix elements $f_{J i}$ remain zero. We further include the orthonormality condition. The Lagrangian thus reads

$$
\begin{aligned}
L= & F_{\mathrm{MP} 2}+F_{\mathrm{R} 12}+\sum_{a i} z_{a i} f_{a i}+\sum_{a I} z_{a I} f_{a I}+\sum_{J i} z_{J i} f_{J i} \\
& -\frac{1}{2} \sum_{r s} x_{r s}\left[\mathbf{C}^{T} \mathbf{S C}-1\right]_{r s},
\end{aligned}
$$

with the Lagrangian multiplier $\mathbf{x}=\mathbf{x}^{\mathrm{T}}$. We define $\overline{\mathbf{z}}=\frac{1}{2}\left(\mathbf{z}+\mathbf{z}^{\mathrm{T}}\right)$ and assume that $z_{I J}=$ $z_{i j}=z_{a b}=z_{i a}=z_{I a}=z_{i J}=0$ and thus $\bar{z}_{a i}=\bar{z}_{i a}=\frac{1}{2} z_{a i}, \bar{z}_{J i}=\bar{z}_{i J}=\frac{1}{2} z_{J i}$ and $\bar{z}_{a I}=\bar{z}_{I a}=\frac{1}{2} z_{a I}$. The Lagrangian is stationary with respect to all wave function parameters except for the integrals, so the energy gradient takes the form

$$
\frac{d E}{d \xi}=\sum_{\mu \nu}\left(\tilde{d}_{\mu \nu} \frac{\partial h_{\mu \nu}}{\partial \xi}-X_{\mu \nu} \frac{\partial S_{\mu \nu}}{\partial \xi}\right)+\sum_{\rho=\{g, r, t\}} \sum_{\mu \nu \kappa \lambda} d_{\mu \nu \kappa \lambda}^{\rho} \frac{\partial\left\langle\mu \nu\left|\hat{O}_{12}^{\rho}\right| \kappa \lambda\right\rangle}{\partial \xi},
$$

where $h_{\mu \nu}$ and $S_{\mu \nu}$ denote the one-electron Hamiltonian nd the overlap integrals, respectively. $\hat{O}_{12}^{\rho}$ can be $r_{12}, r_{12}^{-1}$ or $\left[\hat{T}_{1}, r_{12}\right], \tilde{d}_{\mu \nu}$ are the one-electron density matrix elements and $d_{\mu \nu \kappa \lambda}^{\rho}$ are the elements of the two-electron density matrices. $\mu, \nu \ldots$ denote atomic orbitals, so that $X_{\mu \nu}$ stands for the Lagrangian multipliers $x_{r s}$ in the AO basis. The first term is denoted as the one-electron gradient, the second as the reorthonormalization term and the third one as the two-electron gradient.

\section{The determination of $\mathrm{z}$ and $\mathrm{x}$}

The Lagrangian multipliers are obtained by minimizing the Lagrangian with respect to the molecular orbital coefficients collected in the matrix $\mathbf{C}$. The perturbation of the MO coefficients can be described as

$$
\mathbf{C}(\alpha)=\mathbf{C}(0) \mathbf{U}(\alpha)
$$

collecting the change due to the perturbation in the matrix $\mathbf{U}(\alpha)$. Expanding the Lagrange functional $L$ with respect to $\mathbf{U}$ or $\mathbf{W}=\mathbf{U}-1$ yields

$$
L(\mathbf{W})=L(0)+\operatorname{tr}\left[\mathbf{W}^{T}(\mathbf{A}+\tilde{\mathbf{A}}-\mathbf{x})\right]
$$

where

$$
\tilde{\mathbf{A}}=2\left(\mathbf{f} \overline{\mathbf{z}}+\mathbf{g}(\overline{\mathbf{z}}) \mathbf{d}^{(0)}\right)
$$


is the contribution resulting from the expansion of the Brillouin conditions $f_{i a}=f_{I a}=f_{I j}=$ 0 . The Hartree-Fock density matrices are $d_{i j}^{(0)}=2 \delta_{i j}, d_{I J}^{(0)}=2 \delta_{I J}$, and $d_{a b}^{(0)}=d_{a i}^{(0)}=d_{a I}^{(0)}=$ $d_{i J}^{(0)}=0 . \mathbf{g}(\overline{\mathbf{z}})$ is defines as

$$
[\mathbf{g}(\overline{\mathbf{z}})]_{r s}=\sum_{p q} \bar{z}_{p q}\left\{g_{r s}^{p q}-\frac{1}{2} g_{r p}^{s q}\right\}
$$

Differentiating $L$ with respect to $\mathbf{W}$ at $\mathbf{W}=\mathbf{0}$ yields

$$
\left(\frac{\partial L}{\partial U_{r s}}\right)_{\mathbf{U = 1}}=\left(\frac{\partial L}{\partial W_{r s}}\right)_{\mathbf{W}=\mathbf{0}}=[\mathbf{A}+\tilde{\mathbf{A}}-\mathbf{x}]_{r s}=0 .
$$

From the condition $\mathbf{x}=\mathbf{x}^{T}$ we obtain

$$
\begin{aligned}
x_{r s} & =\frac{1}{2}\left(1+\hat{\tau}_{r s}\right)\left[A_{r s}+\tilde{A}_{r s}\right], \\
0 & =\left(1-\hat{\tau}_{r s}\right)\left[A_{r s}+\tilde{A}_{r s}\right],
\end{aligned}
$$

where $\tau$ is the permutation operator. The first set of equations determines the Lagrangian multipliers $\mathbf{x}$, the second yields the so called Z-vector equations determining the Lagrangian multipliers $\mathbf{z} .^{18}$ The R12 contribution to the Z-vector equations have been discussed in detail in reference 12 .

After having computed $\mathbf{A}$ and solved the Z-vector equations

$$
0=\left(1-\hat{\tau}_{r s}\right)\left[\mathbf{A}+2 \mathbf{f} \overline{\mathbf{z}}+2 \mathbf{g}(\overline{\mathbf{z}}) \mathbf{d}^{(0)}\right]_{r s}
$$

one can evaluate $\tilde{\mathbf{A}}$ and thus can get an expression for $\mathbf{x}$. In the following, we will denote those parts of $\mathbf{x}$ that arise from the evaluation of $\mathbf{A}$ by $\overline{\mathbf{x}}$ and those that arise from the evaluation of $\tilde{\mathbf{A}}$ by $\tilde{\mathbf{x}}$, which implies that $\mathbf{x}=\overline{\mathbf{x}}+\tilde{\mathbf{x}}$. Only the R12 contributions will be given. Details of the construction of $\tilde{\mathbf{A}}$ have been discussed in the course of deriving the effective one-electron density $\tilde{d}_{\mu \nu}$ in reference 12 . For $r s$ being a pair of two inactive occupied orbitals we obtain for $\overline{\mathbf{x}}$ :

$$
\bar{x}_{I J}=\sum_{m n k l q} 4\left(r_{m n}^{I q} g_{J q}^{k l} \tilde{R}_{k l}^{m n}+r_{m n}^{J q} g_{I q}^{k l} \tilde{R}_{k l}^{m n}\right)+2\left(r_{m n}^{I q} t_{J q}^{k l} D_{k l}^{m n}+r_{m n}^{J q} t_{I q}^{k l} D_{k l}^{m n}\right)
$$

In case that $r s$ is a pair of inactive occupied and active occupied orbitals we obtain

$$
\begin{aligned}
\bar{x}_{J i} & =\sum_{m n k l q} 4\left(r_{m n}^{J q} g_{i q}^{k l} \tilde{R}_{k l}^{m n}+r_{m n}^{i q} g_{J q}^{k l} \tilde{R}_{k l}^{m n}\right)+2\left(r_{m n}^{J q} t_{i q}^{k l} D_{k l}^{m n}+r_{m n}^{i q} t_{J q}^{k l} D_{k l}^{m n}\right) \\
& +\sum_{m k l p q} 4\left(r_{m J}^{p q} g_{p q}^{k l} \tilde{R}_{k l}^{m i}+r_{k l}^{p q} g_{p q}^{m J} \tilde{R}_{m i}^{k l}\right)+4 r_{m J}^{p q} t_{p q}^{k l} D_{k l}^{m i}
\end{aligned}
$$




\section{The Two-electron gradient}

\section{Two-electron densities}

For the two-electron gradient we need the derivatives of the integrals $g_{p q}^{m n}, r_{p q}^{m n}$ and $t_{p q}^{m n}$ multiplied with the corresponding two-electron matrices $d_{p m q n}^{\rho}$. In the MO basis they are given as

$$
\begin{aligned}
& d_{p m q n}^{g}=-2 \sum_{k l} \tilde{R}_{m n}^{k l} r_{k l}^{p q} \\
& d_{p m q n}^{r}=-\sum_{k l}\left(D_{m n}^{k l} t_{k l}^{p q}+2 \tilde{R}_{m n}^{k l} g_{p q}^{k l}\right) \\
& d_{p m q n}^{t}=-2 \sum_{k l} D_{m n}^{k l} r_{k l}^{p q}
\end{aligned}
$$

In case that $r s$ is a pair of active virtual orbitals we get

$$
\bar{x}_{a b}=\sum_{m n k l q} 4\left(r_{m n}^{a q} g_{b q}^{k l} \tilde{R}_{k l}^{m n}+r_{m n}^{b q} g_{a q}^{k l} \tilde{R}_{k l}^{m n}\right)+2\left(r_{m n}^{a q} t_{b q}^{k l} D_{k l}^{m n}+r_{m n}^{b q} t_{a q}^{k l} D_{k l}^{m n}\right) .
$$

The intermediates that are needed for the construction of $\mathbf{x}$ are available from the preceding energy calculation and the implementation of $\mathbf{x}$ has been straightforward. Like the implementation of the one- and two-electron terms, it has been accomplished in the DALTON ${ }^{19}$ program.

where $d^{g}{ }_{p m q n}$ is multiplied with the derivatives of $g_{p q}^{m n}, d^{r}{ }_{p m q n}$ with the derivatives of $r_{p q}^{m n}$ and $d_{p m q n}^{t}$ the derivatives of with $t_{p q}^{m n}$. 


\section{Integral derivatives}

Since the transformation of the two-electron integrals to contracted solid-harmonic basis functions can be carried out in a separate step, we begin with differentiating the following Cartesian Gaussian-type orbitals (GTO's) with respect to the position of a nucleus $N$ :

$$
\frac{d(\alpha \beta|\hat{O}| \gamma \delta)}{d N_{\xi}}=\delta_{A N} \frac{d(\alpha \beta|\hat{O}| \gamma \delta)}{d A_{\xi}}+\delta_{B N} \frac{d(\alpha \beta|\hat{O}| \gamma \delta)}{d B_{\xi}}+\delta_{C N} \frac{d(\alpha \beta|\hat{O}| \gamma \delta)}{d C_{\xi}}+\delta_{D N} \frac{d(\alpha \beta|\hat{O}| \gamma \delta)}{d D_{\xi}}
$$

where $\xi=\{x, y, z\}$ is a Cartesian direction. $\alpha, \beta, \gamma, \delta$ indicate Cartesian Gaussians in the usual form ${ }^{20}$

$$
\alpha \equiv G_{i j k}(\mathbf{r}, a, \mathbf{A})=\left(x-A_{x}\right)^{i}\left(y-A_{y}\right)^{j}\left(z-A_{z}\right)^{k} e^{-a\left[\left(x-A_{x}\right)^{2}+\left(y-A_{y}\right)^{2}+\left(z-A_{z}\right)^{2}\right]} .
$$

$\hat{O}$ is one of the following operators

$$
\frac{1}{r_{12}}, \quad r_{12}, \quad\left[\hat{T}_{1}, r_{12}\right] \equiv\left[-\frac{1}{2} \nabla_{1}^{2}, r_{12}\right]
$$

Following the McMurchie-Davidson scheme, ${ }^{20}$ a product of Cartesian Gaussians is expanded exactly in the Hermite polynomials $\Lambda_{t u v}$ as follows

$$
G_{i j k}(\mathbf{r}, a, \mathbf{A}) G_{l m n}(\mathbf{r}, b, \mathbf{B})=\sum_{t=0}^{i+l} \sum_{u=0}^{j+m} \sum_{v=0}^{k+n} E_{t}^{i l ; 0} E_{u}^{j m ; 0} E_{v}^{k n ; 0} \Lambda_{t u v}(\mathbf{r}, p, \mathbf{P})
$$

where

$$
\mathbf{P}=\frac{a}{p} \mathbf{A}+\frac{b}{p} \mathbf{B} \quad \text { and } \quad p=a+b .
$$

Based on this expansion and on the simple relation

$$
\frac{d G_{i j k}}{d A_{x}}=2 a G_{i+1, j, k}-i G_{i-1, j, k}
$$

codes for the evaluation of two-electron integrals over the operator $r_{12}^{-1}$ and its first and higher derivatives have been available in the DALTON ${ }^{19}$ program for many years. The calculation of the derivatives of the integrals over $r_{12}$ can be carried out in an analogous manner, and their implementation was completely straightforward. The computation of integrals over the operator $\left[\hat{T}_{1}, r_{12}\right]$ had not been carried out before and was accomplished in the course of the present work. The undifferentiated commutator integrals are computed according to reference 21

$$
\left(\alpha \beta\left|\left[\hat{T}_{1}, r_{12}\right]\right| \gamma \delta\right)=\left(\frac{b-a}{a+b}\right)\left(\alpha \beta\left|r_{12}^{-1}\right| \gamma \delta\right)-\nabla_{P} \nabla_{R}\left(\alpha \beta\left|r_{12}\right| \gamma \delta\right),
$$


where $\mathbf{R}=\mathbf{A}-\mathbf{B}$ and

$$
\frac{\partial}{\partial A_{x}}=\frac{a}{p} \frac{\partial}{\partial P_{x}}+\frac{\partial}{\partial R_{x}} .
$$

Since the Hermite functions depend only on $P_{x}$

$$
\frac{\partial \Lambda_{t}}{\partial P_{x}}=\Lambda_{t+1}
$$

and the expansion coefficients only on $R_{x}$

$$
\frac{\partial E_{t}^{i l ; n}}{\partial R_{x}}=E_{t}^{i l ; n+1},
$$

we obtain

$$
\begin{aligned}
& \frac{\partial}{\partial A_{x}}\left[\nabla_{P} \nabla_{R} \sum_{t=0}^{i+l} \sum_{u=0}^{j+m} \sum_{v=0}^{k+n} E_{t}^{i l ; 0} E_{u}^{j m ; 0} E_{v}^{k n ; 0}\left(\Lambda_{t u v}\left|r_{12}\right| \gamma \delta\right)\right] \\
= & \sum_{t=0}^{i+l} \sum_{u=0}^{j+m} \sum_{v=0}^{k+n}\left[\frac{a}{p} E_{t}^{i l ; 1} E_{u}^{j m ; 0} E_{v}^{k n ; 0}\left(\Lambda_{t+2, u, v}\left|r_{12}\right| \gamma \delta\right)+E_{t}^{i l ; 2} E_{u}^{j m ; 0} E_{v}^{k n ; 0}\left(\Lambda_{t+1, u, v}\left|r_{12}\right| \gamma \delta\right)\right. \\
+ & \frac{a}{p} E_{t}^{i l ; 0} E_{u}^{j m ; 1} E_{v}^{k n ; 0}\left(\Lambda_{t+1, u+1, v}\left|r_{12}\right| \gamma \delta\right)+E_{t}^{i l ; 1} E_{u}^{j m ; 1} E_{v}^{k n ; 0}\left(\Lambda_{t, u+1, v}\left|r_{12}\right| \gamma \delta\right) \\
+ & \left.\frac{a}{p} E_{t}^{i l ; 0} E_{u}^{j m ; 0} E_{v}^{k n ; 1}\left(\Lambda_{t+1, u, v+1}\left|r_{12}\right| \gamma \delta\right)+E_{t}^{i l ; 1} E_{u}^{j m ; 0} E_{v}^{k n ; 1}\left(\Lambda_{t, u, v+1}\left|r_{12}\right| \gamma \delta\right)\right] .
\end{aligned}
$$

Thus, the derivatives of integrals over $\left[\hat{T}_{1}, r_{12}\right]$ can be calculated from integrals over $r_{12}^{-1}$ and $r_{12}$ over Hermite functions. The additional costs with respect to the computation of derivatives of integrals over $r_{12}$ result from the fact that the maximum angular momentum of the calculated $r_{12}$ integrals over Hermite functions must be increased by one. Moreover, while for the computation of undifferentiated integrals over $\left[\hat{T}_{1}, r_{12}\right]$ just the coefficients $E^{i l ; 0}$ and $E^{i l ; 1}$ are needed, derivatives of integrals over the operator $\left[\hat{T}_{1}, r_{12}\right]$ require second derivatives of the expansion coefficients $\left(E^{i l ; 2}\right)$. These can be easily calculated by using the recursion formula

$$
E_{t}^{i+1, j ; n}=\frac{1}{2 p} E_{t-1}^{i j ; n}-\frac{b}{p}\left(R_{x} E_{t}^{i j ; n}+n E_{t}^{i j ; n-1}\right)+(t+1) E_{t+1}^{i j ; n}
$$

with starting values

$$
E_{0}^{00 ; 0}=\exp \left(-\frac{a b}{p} R_{x}^{2}\right) \quad \text { and } \quad E_{0}^{00 ; n+1}=-\frac{2 a b}{p}\left(R_{x} E_{0}^{00 ; n}+n E_{0}^{00 ; n-1}\right) .
$$

For the calculation of the derivatives with respect to the displacement of the nucleus $B$, similar formulas can be derived, by considering that

$$
\frac{\partial}{\partial B_{x}}=\frac{b}{p} \frac{\partial}{\partial P_{x}}-\frac{\partial}{\partial R_{x}} .
$$


Thus we get for the displacement of the nuclei $C$ and $D$

$$
\begin{aligned}
& \frac{\partial}{\partial C_{x}}\left[\nabla_{P} \nabla_{R} \sum_{t=0}^{i+l} \sum_{u=0}^{j+m} \sum_{v=0}^{k+n} E_{t}^{i l ; 0} E_{u}^{j m ; 0} E_{v}^{k n ; 0}\left(\Lambda_{t u v}\left|r_{12}\right| \gamma \delta\right)\right] \\
= & \sum_{t=0}^{i+l} \sum_{u=0}^{j+m} \sum_{v=0}^{k+n}\left[E_{t}^{i l ; 1} E_{u}^{j m ; 0} E_{v}^{k n ; 0}\left(\Lambda_{t+1, u v}\left|r_{12}\right|\left(\frac{\partial \gamma}{\partial C_{x}}\right) \delta\right)\right. \\
+ & \left.E_{t}^{i l ; 0} E_{u}^{j m ; 1} E_{v}^{k n ; 0}\left(\Lambda_{t, u+1, v}\left|r_{12}\right|\left(\frac{\partial \gamma}{\partial C_{x}}\right) \delta\right)+E_{t}^{i l ; 0} E_{u}^{j m ; 0} E_{v}^{k n ; 1}\left(\Lambda_{t, u, v+1}\left|r_{12}\right|\left(\frac{\partial \gamma}{\partial C_{x}}\right) \delta\right)\right] .
\end{aligned}
$$

The implementation of the two-electron terms follows the one described in reference 22 .

\section{APPLICATIONS}

As an application, geometry optimizations of nine stationary points on the potential energy surface of the water dimer have been performed at the level of MP2-R12 using the analytical gradients introduced in the last section.

Our calculations are based on a selection of structures, which have been characterized by Smith et $a l .{ }^{15}$ as stationary points on the potential surface of the water dimer. In an unpublished study, van Duijneveldt et al. ${ }^{13}$ found another structure (structure 11 in figure 1), which has been included in our study. Only structures that correspond to a minimum within a prescribed point group symmetry have been considered and structures 2 and 7 have therefore been omitted. All optimized structures are shown in figure 1. The numbers correspond to those of earlier studies ${ }^{14,15}$ and have been retained to avoid confusion. We will compare our results with those obtained by Smith et al., who calculated energies and vibrational frequencies at the level of MP2/6-31G(d,p) and MP2/6-311+G(d,p), Tschumper et al., who did these calculations at the level of $\operatorname{CCSD}(\mathrm{T}) / \mathrm{TZ} 2 \mathrm{P}(f, d)+$ dif and van Duijneveldt at al., who used counterpoise corrected MP2 in an IOM basis set. The latter contains $5 \mathrm{~s}, 3 \mathrm{p}, 3 \mathrm{~d}, 2 \mathrm{f}, 1 \mathrm{~g}$-functions for $\mathrm{O}$ and $2 \mathrm{~s}, 2 \mathrm{p}$ functions for $\mathrm{H}$ and was optimized for the methanol dimer. ${ }^{23}$ In all three studies geometries optimized at the corresponding level of theory were used. Van Duijneveldt et al. fixed the geometries of the monomers at an O-H distance of $0.9573 \AA$ and an angle of $104.52^{\circ}$, whereas Smith et al. and Tschumper et al. performed full geometry optimizations. According to experimental and theoretical studies ${ }^{14,15,24-26}$ structure 1 is the minimum structure, whereas according to references 14 and 15, structures 2, 
4 and 9 are transition states. The rest of the species corresponds to stationary points of higher order. ${ }^{14,15}$

\section{A. Computational Details}

For each stationary point full geometry optimizations were performed in the point group of each structure. Each calculation was done with the program package DALTON. ${ }^{19}$ Two orbitals have been frozen within the frozen core approximation. For $\mathrm{O}$ a (16s9p5d4f2g/8s7p5d4f2g) basis was used, which consists of the QZV basis of Schäfer et $a l .,{ }^{27}$ augmented by diffuse s- and p-functions from Dunning's aug-cc-pVQZ ${ }^{28,29}$ basis. The p-basis has been partially decontracted. All of the d-, f- and g-functions from Dunning's aug-cc-pVQZ basis as well as tight d- (11.0) and f- (8.27) functions have been added. For $\mathrm{H}$ a $(8 \mathrm{~s} 4 \mathrm{p} 3 \mathrm{~d} 2 \mathrm{f} / 6 \mathrm{~s} 4 \mathrm{p} 3 \mathrm{~d} 2 \mathrm{f})$ basis was used. Like the basis for O it consists of the QZV basis of Schäfer et al. ${ }^{27}$ augmented by diffuse s-functions as well as p-, d- and f-functions from Dunning's aug-cc-pVQZ basis. The set of s-functions has been partially decontracted. Except for structures 8 and 11 the convergence threshold for the change in energy during the geometry optimization was the maximum of $1.0 \cdot 10^{-6}$ Hartree and two times the convergence threshold for the wave function, whereas for the norm of the gradient and step it was the maximum of $1.0 \cdot 10^{-5}$ a.u. and two times the convergence threshold for the wave function. Two out of these three criteria had to decrease below their threshold before the program declared convergence. For structures 8 and 11 the somewhat looser convergence criteria of Baker $^{30}$ were applied.

\section{B. Results and discussion}

\section{Geometries}

The optimized geometrical parameters determined at the level of MP2-R12 theory in the basis described above are given in tables I and II for the intramonomer and the intermonomer parameters respectively. To facilitate the reproduction of the data some redundant parameters have been included. In table III some of the parameters are shown together with the corresponding values from references 15, 14 and 13. To give a better understanding of the effects due to the chosen basis and method on the geometries, we will first compare the 
results of the latter three studies.

The study of van Duijneveldt et al. was motivated by the somewhat inconsistent picture that arises when one compares the bond distances obtained by Smith et al. with those of Tschumper et al.. While the study of Tschumper et al. gives longer O-O bond distances for most structures $(0.08 \AA$ for structures 9 and 10$)$, the O-O bond distance of structure 8 is $0.09 \AA$ shorter than in the study of Smith et al.. Van Duijneveldt et al. assumed that this is due to a more or less erratic compensation of a smaller basis set superposition error (BSSE) in the $\operatorname{TZ2} P(f, d)+$ dif basis resulting in lengthenings of the bond distances and a greater dispersion attraction in this basis resulting in a shortening of the bond distances. Therefore they performed geometry optimizations at the level of counterpoise corrected MP2 avoiding the shortenings due to BSSE. The IOM should also allow for a better description of the dispersion attraction. The resulting bond distances were stretched compared to those of reference 14. For structures 1 to 6 the differences amount to ca. $0.02 \AA$, for the more weakly bound structures 8 to 10 the deviations are larger (up to $0.1 \AA$ ). There are several possible reasons for these deviations. First of all the MP2/IOM calculations underestimate the dispersion attraction by ca. $10 \%$ yielding too large O-O bond distances. On the other hand there is an unknown error cancellation of the BSIE and the BSSE in the study of Tschumper et al., which could lead to shortenings of the bond distances. Another reason could be the method itself. The interaction energies, which will be discussed in the next section, give another indication for this.

To study this in greater detail van Duijneveldt et al. optimized the O-O distance of structures 1,8 and 9 at different levels of theory with and without counterpoise correction. Structure 1 is representative for structures 1 to 6 , where the deviations between the two studies are rather small, whereas structures 8 and 9 are cases where larger deviations occur. The results are shown in table IV. Column 3 shows the deviations at the level of counterpoise corrected $\operatorname{CCSD}(\mathrm{T})$. There is almost no difference for structure 1 , whereas the O-O distances are shortened for structures 8 and 9. This parallels the influence of $\operatorname{CCSD}(\mathrm{T})$ on the interaction energies. Nevertheless this effect is not large enough to explain the short bond distances in the study of Tschumper et al.. One explanation is that these are shortened by BSSE. This is supported by the results in columns 3 and 4 . The combination of the O-O distances without counterpoise correction with those obtained at the level of CCSD(T) give even shorter values than those obtained by Tschumper et al.. The reason for this is probably 
the smaller BSSE in the TZ2P $(d, f)+$ dif basis. The effect of increasing the basis set to the basis set limit at the level of MP2 is shown in column 6. Combining these effects with those resulting from the use of $\operatorname{CCSD}(\mathrm{T})$ (column 3), one gets the best ab initio O-O distances shown in column 7 . For structure 1 there is a good agreement between this value and the one obtained at MP2/IOM/CP level of theory, whereas for structures 8 and 9 the O-O distances are elongated by $0.05 \AA$ and $0.1 \AA$ respectively. The values obtained by Tschumper et al. agree due to error compensation very well with the best ab initio O-O distances. It is quite probable that this applies for the other structures as well.

Compared to the values given by Tschumper et al. our results show smaller deviations (max. $0.017 \AA$ for $r_{\mathrm{O}_{1} \mathrm{O}_{2}}$ in structure 5 and $0.05 \AA$ for $r_{\mathrm{O}_{1} \mathrm{O}_{2}}$ in structure 8) than those that have been found in the comparison of the other two studies. The deviation of the intramonomer parameters are even smaller amounting to ca. $0.005 \AA$. Compared to the bond lengths of the free monomer the intramonomer bond lengths of structures 8 to 10 are slightly stretched, whereas the parameters $r_{\mathrm{O}_{1} \mathrm{H}_{1}}$ are slightly shortened for strucutures 1 to 6 and the parameters $r_{\mathrm{O}_{1} \mathrm{H}_{2}}$ are up to $0.0075 \AA$ larger. As it is obvious from figure 1 the latter distances are stretched due to hydrogen bridges to the other monomer. The stronger the respective structure is bound, the more this is distance is stretched. As expected the largest deviation occurs for the ground state $(0.0075 \AA)$. In parallel to the interaction energy this distance decreases in table I from top to bottom for structures 1 to 6 . The deviations to the corresponding distance of the free monomer are smaller for structures 8 to 10, which could be as well due to the larger distance of the two monomers in these structures as for symmetry reasons.

The intramonomer bond angles differ only slightly from those of the free monomer for structures 1 to 6 , whereas they deviate by up to $2.3^{\circ}$ to smaller values for structures 8 to 10 . This could be due to the hydrogen bonds as well, which cause a reduction of the bond angles and thus a decrease of the respective bond distances, as it is obvious from figure 1.

Let's turn to our results obtained at the MP2-R12 level of theory in the modified QZV basis. The intermolecular parameters differ from those found by Tschumper et al. to a larger extent than the intramolecular ones. The maximum deviation amounts to $0.017 \AA$ for the O-O distance of structure 5 and $0.05 \AA$ for the same distance in structure $8 . r_{\mathrm{H}_{2} \mathrm{O}_{2}}$ und $r_{\mathrm{O}_{1} \mathrm{O}_{2}}$ are shortened for structures 1 to 6 , whereas they are stretched for the more weakly bound structures 8 to $10\left(0.05 \AA\right.$ and $0.007 \AA$ for $r_{\mathrm{O}_{1} \mathrm{O}_{2}}$ in structures 8 and 9$)$ or of the same 
length $\left(r_{\mathrm{O}_{1} \mathrm{O}_{2}}\right.$ in structure 10). These effects could be due to the CCSD(T) method itself, which possibly decribes the bond between the two monomers in structures 8 to 10 better than MP2. In contrast the MP2/IOM bond lengths of van Duijneveldt et al. are in general stretched compared to our results. The deviations with respect to the values obtained by Smith et al. correspond to those between the results of Tschumper et al. and Smith et al.. We will now consider the values of table IV once again. First of all the MP2-R12 bond lengths obtained by van Duijneveldt et al. are stretched compared to our values. For structure 1 the deviation is relatively small $(0.005 \AA)$, for structures 8 and 9 it is larger with up to ca. $0.016 \AA$. This is probably due to the fact that van Duijneveldt et al. optimized only the O-O distance whereas we performed a full geometry optimization. It is difficult to estimate this effect on the geometries of the different structures. According to figure 1 one can imagine that it has less effect on structure 1 than on structure 9 and 10. The differences could also be due to the smaller basis that van Duijneveldt et al. used in their study, but this effect affects the geometries probably only slightly. For structures 1 and 9 our MP2-R12 results are in good agreement with the results of Tschumper et al. and with the best ab initio values in column $7 .{ }^{13}$ The deviation between our values and those of Tschumper et al. and van Duijneveldt et al. for structure 8 could be due to the fact, that the CCSD(T) correction is of greater importance for this structure than for the other two structures.

The bond angles $\theta_{\mathrm{O}_{1} \mathrm{H}_{2} \mathrm{O}_{2}}$ are in general smaller (1.9 ${ }^{\circ}$ for structure 3$)$ in the MP2-R12 optimized structures compared to those of reference 14. An exception is structure 8. Here $\theta_{\mathrm{O}_{1} \mathrm{H}_{2} \mathrm{O}_{2}}$ is larger by $0.042^{\circ}$ compared to the $\operatorname{CCSD}(\mathrm{T})$ optimized geometry. For the rest of the angles no tendency can be found, they differ to a relatively large amount, the max. deviation for a bond angle being $2.9^{\circ}\left(\theta_{\mathrm{H}_{3} \mathrm{O}_{2} \mathrm{O}_{1}}\right.$ in structure 5$)$ and the max. deviation for a dihedral being $4.9^{\circ}\left(\tau_{\mathrm{H}_{3} \mathrm{O}_{2} \mathrm{O}_{1} \mathrm{H}_{2}}\right.$ in structure 5$)$. These differences have relatively little affect on the relative energies due to the flat potential energy surface along these amplitudes. ${ }^{14}$

\section{Energies}

The dissociation energies for the considered structures calculated at different levels of theory are shown in table V. Columns 2 to 5 show results obtained with MP2, CCSD, CCSD(T) and MP2-R12 from the study of van Duijneveldt et al.. Here the geometries optimized at the level of counterpoise corrected MP2/IOM were used. To get an insight 
on how the choice of basis and method affects the dissociation energies, we will start our consideration with a comparison of these results.

In all three cases there is a significant difference between the way the improved method influences structures 1 to 6 and the way it influences structures 8 to 10. Going from MP2 to CCSD $\Delta E$ decreases by $0.7 \mathrm{~kJ} / \mathrm{mol}$ for structures 1 to 6 , whereas there is almost no effect or a slight increase for structures 8 to 10 . As already mentioned structures 8 to 10 differ from structures 1 to 6 by a longer O-O distance. At the level of optimized MP2/IOM/CP it amounts to $2.79 \AA$ to $2.94 \AA$ for structures 1 to 6 , whereas is amounts to $3.06 \AA$ to $3.39 \AA$ for structures 8 to 10 . If one goes further to $\operatorname{CCSD}(\mathrm{T})$, one finds an increase of $\Delta E$ for all species. This effect is larger for structures 1 to 6 than for structures 8 to 10 . Overall the transition from MP2 to $\operatorname{CCSD}(\mathrm{T})$ has only a slight effect on the first set of structures, whereas $\Delta E$ increases by about $0.5 \mathrm{~kJ} / \mathrm{mol}$ for the second set of structures. From figure 1 we see that for structures 8 to 10 more electrons take part in the hydrogen bonds than for structures 1 to 6 , so possibly $\operatorname{CCSD}(\mathrm{T})$ decribes the bond situation in these structures more adequately than MP2 by introducing triple excitations. The MP2-R12 result reflects the effect of an increase of the basis set. Compared to the MP2 results all values are increased by about $10 \%$. Combining all possible improvements one obtains the results in column 7 . These energies are larger than those obtained at the level of MP2/IOM/CP, but the relative energies of structures 2 to 10 compared to structure 1 are almost identical for both columns. The dissociation energies calculated at the level of MP2-R12 are larger for the MP2-R12 optimized structures compared to those optimized at the level of the MP2/IOM/CP (column 5). The largest deviation occurs for structure $9(0.51 \mathrm{~kJ} / \mathrm{mol})$. The reasons for this are probably the different geometries these results were obtained at. Another, probably small effect could be due to the larger basis set used for the calculation of the energies in column 9.

Comparing the dissociation energies obtained for the MP2-R12 optimized structures with the best ab initio values of van Duijneveldt et al. (column 6), one finds larger dissociation energies for structure 1 to 6 (up to $0.23 \mathrm{~kJ} / \mathrm{mol}$ for structure 1 ), whereas for structures 8 to 10 the dissociation energies are smaller (up to $0.36 \mathrm{~kJ} / \mathrm{mol}$ for structure 8 ). The dissociation energy of structure 9 is $0.05 \mathrm{~kJ} / \mathrm{mol}$ below the best ab initio values of van Duijneveldt et al. $\Delta E_{\text {korr. }}$ In general our MP2-R12 results agree better with the best ab initio values of van Duijneveldt et al. than with their MP2-R12 values shown in column 5 . It is difficult to 
give a reason for this, since it is not possible to seperate the many different effects playing a role in these calculations in the course of our work, e.g. the fixed monomer geometries of reference 13 .

The MP2-R12 results obtained by Tschumper et al. are shown in column 7 . They were calculated at the geometries optimized with $\operatorname{CCSD}(\mathrm{T})$ in a $\operatorname{TZ2P}(f, d)+$ dif basis. A K2 named basis was used which was derived from Dunning's cc-pV5Z basis. The resulting dissociation energies agree very well with our values, the maximum deviation amounts to $0.13 \mathrm{~kJ} / \mathrm{mol}$ for structure 3. This agreement is probably due to the fact that the geometries are very similar and that both basis sets are close to the basis set limit.

Column 8 shows the best results of Tschumper et al. obtained for the $\operatorname{CCSD}(\mathrm{T}) / \mathrm{TZ2} \mathrm{P}(f, d)+$ dif optimized geometries including corrections for the BSIE, core correlation, quadruple excitations and relativistic effects. Our results are quite close to these values, yielding in most cases smaller dissociation energies with maximum deviations of $0.42 \mathrm{~kJ} / \mathrm{mol}$ for structure 9 and $0.51 \mathrm{~kJ} / \mathrm{mol}$ for structure 8 . For structure 3 we obtain a larger dissociation energy $(0.08 \mathrm{~kJ} / \mathrm{mol})$. In general the deviations for structures 1 to 6 are smaller than for structures 8 to 10, whereas larger $\operatorname{CCSD}(\mathrm{T})$ values occur for the latter set of structures compared to MP2. This is probably the effect of the method CCS(T) itself.

Finally we consider the characteristics of structure 11. With a dissociation energy of $2.54 \mathrm{~kJ} / \mathrm{mol}$ it is very weakly bound. The bond distances and bond angles hardly differ from the values of the free monomer. The O-O distance is very long $(3.879 \AA)$, giving another indication for the very weak bond.

\section{FINAL REMARK}

In the present paper we have presented analytical gradients for the MP2-R12/A method. They can be constructed from the corresponding Lagrangian functional requiring the derivatives of the integrals over $r_{12}^{-1}, r_{12}$ and $\left[\hat{T}_{1}, r_{12}\right]$. The intermediates needed for building the reorthonormalization term and the two-electron densities are available from the preceding energy calculation. The construction of the effective one-electron density has been discussed in detail in reference 12 .

As a test calculation we have performed geometry optimizations of nine stationary points on the potential energy surface of the water dimer. The results have been compared with those 
1 E. A. Hylleraas, Z. Phys., 54, 347 (1929).

2 W. Klopper and W. Kutzelnigg, Chem. Phys. Lett. 134, 17 (1987).

3 W. Klopper, J. Chem. Phys. 120, 10890 (2004).

4 S. Kedzuch, M. Milko and J. Noga, Int. J. Quantum Chem. 105, 929, 2005

${ }^{5}$ F. R. Manby, J. Chem. Phys. 119, 4607 (2003).

6 E. F. Valeev, Chem. Phys. Lett. 395, 190 (2004).

7 S. Ten-no, Chem. Phys. Lett. 398, 56 (2004).

8 D. P. Tew and W. Klopper, J. Chem. Phys. 123, 74101 (2005).

9 E. F. Valeev and C. L. Janssen, J. Chem. Phys. 121, 1214 (2004).

10 A. Glöß, C. Villani and W. Klopper, unpublished.

11 H. Fliegl, W. Klopper and C. Hättig, J. Chem. Phys. 124, 044112 (2006).

12 E. Kordel and C. Villani and W. Klopper, J. Chem. Phys. 122, 214306 (2005).

13 J. G. C. M. van Duijneveldt-van de Rijdt, F. B. van Duijneveldt, W. T. M. Mooij, W. Klopper and P. J. A. Ruttink, 2003, unpublished.

14 G. S. Tschumper, M. L.Leininger, B. C. Hoffmann, E. F. Valeev, H. F. Schaefer III and M. Quack, J. Chem. Phys. 116, 690 (2002).

15 B. J. Smith, D. J. Swanton, J. A. Pople, H. F. Schaefer III and L. Radom, J. Chem. Phys. 92, $1240(1990)$.

16 W. Klopper, C. C. M. Samson, J. Chem. Phys. 116, 6297 (2002). 
17 M. Schütz, H.-J. Werner, R. Lindh and F. R. Manby, J. Chem. Phys. 121, 737 (2004).

18 N. C. Handy, H. F. Schaefer III, J. Chem. Phys. 81, 5031 (1994).

19 T. Helgaker, H. J. Aa. Jensen, P. Jørgensen et al. DALTON, a molecular electronic structure program.

20 L. E. McMurchie and E. R. Davidson, J. Comp. Phys. 26, 218 (1978).

21 W. Klopper and R. Röhse, Theor. Chim. Acta 83, 441 (1992).

22 K. Hald, A. Halkier, P. Jørgensen, S. Coriani, C. Hättig and T. Helgaker, J. Chem. Phys. 118, 2985 (2003).

23 W. T. M. Mooij, F. B. van Duijneveldt, J. G. C. M. van Duijneveldt-van de Rijdt and B. P. van Eijck, J. Phys. Chem. A, 103, 9872 (1999).

24 T. R. Dyke, J. Chem. Phys. 66, 492 (1977).

25 T. R. Dyke, K. M. Mack and J. S. Muenter, J. Chem. Phys. 66, 498 (1977).

26 J. A. Odutola and T. R. Dyke, J. Chem. Phys. 72, 5062 (1980).

27 A. Schäfer, C. Huber and R. Ahlrichs, J. Chem. Phys. 100, 5829 (1994).

28 R. A. Kendall, T. H. Dunning, Jr., and R. J. Harrison, J. Chem. Phys. 96, 6796 (1992).

29 T. H. Dunning, J. Chem. Phys. 90, 1007 (1989).

30 J. Baker, J. Comput. Chem., 14, 1085 (1993).

31 P. Pulay, Mol. Phys. 17, 197 (1969). 
TABLE I: Intramolecular parameters of $\mathrm{H}_{2} \mathrm{O}$ and $\left(\mathrm{H}_{2} \mathrm{O}\right)_{2}$ optimized at the MP2-R12 level in an augmented QZV basis. Bond lengths are in $\AA$, angles $\theta_{X Y Z}$ are in deg. The values in parantheses correspond to those of reference 14. The atom numbers correspond to those depicted in Fig. 1

\begin{tabular}{|c|c|c|c|c|c|c|}
\hline Struktur & $r_{\mathrm{O}_{1} \mathrm{H}_{1}}$ & $r_{\mathrm{O}_{1} \mathrm{H}_{2}}$ & $r_{\mathrm{O}_{2} \mathrm{H}_{3}}$ & $r_{\mathrm{O}_{2} \mathrm{H}_{4}}$ & $\theta_{\mathrm{H}_{1} \mathrm{O}_{1} \mathrm{H}_{2}}$ & $\theta_{\mathrm{H}_{3} \mathrm{O}_{1} \mathrm{H}_{4}}$ \\
\hline \multirow[t]{2}{*}{ Monomer } & 0.9586 & 0.9586 & $\cdots$ & $\cdots$ & 104.4 & $\cdots$ \\
\hline & $(0.9589)$ & $(0.9589)$ & $\cdots$ & $\cdots$ & (104.16) & . \\
\hline \multirow[t]{2}{*}{1} & 0.9576 & 0.9661 & 0.9595 & 0.9595 & 104.76 & 104.83 \\
\hline & $(0.9581)$ & $(0.9653)$ & $(0.9597)$ & $(0.9597)$ & $(104.45)$ & $(104.58)$ \\
\hline \multirow[t]{2}{*}{3} & 0.9575 & 0.9647 & 0.9589 & 0.9583 & 104.81 & 105.31 \\
\hline & $(0.9579)$ & $(0.9640)$ & $(0.9590)$ & $(0.9585)$ & $(104.48)$ & (105.04) \\
\hline \multirow[t]{2}{*}{4} & 0.9583 & 0.9619 & 0.9583 & 0.9619 & 105.13 & 105.13 \\
\hline & $(0.9585)$ & $(0.9616)$ & $(0.9586)$ & $(0.9616)$ & $(104.84)$ & $(104.84)$ \\
\hline \multirow[t]{2}{*}{5} & 0.9580 & 0.9616 & 0.9580 & 0.9616 & 105.24 & 105.24 \\
\hline & $(0.9583)$ & $(0.9614)$ & $(0.9583)$ & $(0.9614)$ & $(104.95)$ & $(104.95)$ \\
\hline \multirow[t]{2}{*}{6} & 0.9578 & 0.9613 & 0.9578 & 0.9613 & 105.37 & 105.37 \\
\hline & $(0.9580)$ & $(0.9611)$ & $(0.9580)$ & $(0.9611)$ & $(105.14)$ & (105.14) \\
\hline \multirow[t]{2}{*}{8} & 0.9592 & 0.9592 & 0.9592 & 0.9592 & 103.32 & 103.32 \\
\hline & $(0.9594)$ & $(0.9594)$ & $(0.9594)$ & $(0.9594)$ & $(103.15)$ & $(103.15)$ \\
\hline \multirow[t]{2}{*}{9} & 0.9591 & 0.9591 & 0.9595 & 0.9595 & 101.67 & 104.63 \\
\hline & $(0.9596)$ & $(0.9596)$ & $(0.9593)$ & $(0.9593)$ & $(101.56)$ & $(104.42)$ \\
\hline \multirow[t]{2}{*}{10} & 0.9590 & 0.9590 & 0.9590 & 0.9590 & 102.02 & 104.28 \\
\hline & $(0.9591)$ & $(0.9591)$ & $(0.9592)$ & $(0.9592)$ & (101.91) & (104.09) \\
\hline 11 & 0.9586 & 0.9586 & 0.9587 & 0.9587 & 105.31 & 105.31 \\
\hline
\end{tabular}




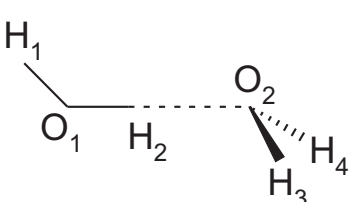

$1 \mathrm{C}_{\mathrm{s}}$<smiles></smiles>

$4 C_{i}$<smiles>CC(=O)CCO</smiles>

$6 \mathrm{C}_{2 \mathrm{~h}}$ planar

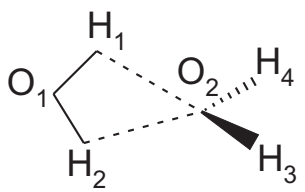

$9 \mathrm{C}_{2 \mathrm{v}}$<smiles>COCCO</smiles>

$3 \mathrm{C}_{\mathrm{s}}$ planar<smiles>CC1CC(O)C1</smiles>

$5 \mathrm{C}_{2}$

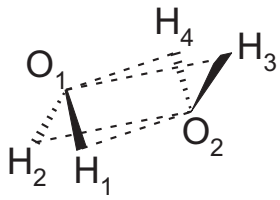

$8 C_{2 h}$<smiles>CC1(C)[CH]OC1</smiles>

$10 C_{2 v}$ planar

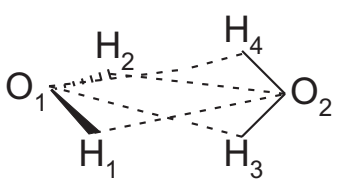

$11 \mathrm{C}_{2 \mathrm{v}}$

FIG. 1: The structures of the 9 stationary points optmized in this study. 
TABLE II: Intermolecular parameters of $\left(\mathrm{H}_{2} \mathrm{O}\right)_{2}$ optimized at the MP2-R12 level in an augmented QZV basis. Bond lengths are in $\AA$ and angles in degrees. The values in parentheses correspond to those of reference 14. The atom numbers correspond to those depicted in Fig. 1

\begin{tabular}{|c|c|c|c|c|c|c|c|}
\hline Struk & $r_{\mathrm{O}_{2} \mathrm{H}_{2}}$ & $\theta_{\mathrm{O}_{1} \mathrm{H}_{2} \mathrm{O} 2}$ & $\theta_{\mathrm{H}_{3} \mathrm{O}_{2} \mathrm{O}_{1}}$ & $\theta_{\mathrm{H}_{4} \mathrm{O}_{2} \mathrm{O}_{1}}$ & $\tau_{\mathrm{O}_{2} \mathrm{H}_{2} \mathrm{O}_{1} \mathrm{H}_{1}}$ & $\tau_{\mathrm{H}_{3} \mathrm{O}_{2} \mathrm{O}_{1} \mathrm{H}_{2}}$ & $\tau_{\mathrm{H}_{4} \mathrm{O}_{2} \mathrm{O}_{1} \mathrm{H}_{2}}$ \\
\hline \multirow[t]{2}{*}{1} & 1.9474 & 171.68 & 110.41 & 110.41 & 180.00 & 122.27 & -122.27 \\
\hline & $(1.9485)$ & $(172.92)$ & $(119.50)$ & $(110.50)$ & $(180.00)$ & (122.37) & $(-122.37)$ \\
\hline \multirow[t]{2}{*}{3} & 1.9714 & 165.69 & 109.50 & 145.19 & 180.00 & 180.00 & 0.00 \\
\hline & (1.9813) & $(167.59)$ & (109.96) & $(145.00)$ & $(180.00)$ & $(180.00)$ & $(0.00)$ \\
\hline \multirow[t]{2}{*}{4} & 2.2702 & 114.35 & 134.64 & 47.46 & 137.71 & 114.10 & 180.00 \\
\hline & $(2.2796)$ & (114.84) & $(132.32)$ & $(47.15)$ & $(134.78)$ & $(111.86)$ & 180.00 \\
\hline \multirow[t]{2}{*}{5} & 2.2729 & 111.79 & 147.98 & 49.45 & -157.41 & -123.18 & 167.51 \\
\hline & $(2.2810)$ & $(112.52)$ & $(145.10)$ & (48.95) & $(-153.28)$ & $(-118.27)$ & $(-167.68)$ \\
\hline \multirow[t]{2}{*}{6} & 2.2745 & 109.98 & 156.25 & 50.88 & 180.00 & 180.00 & 180.00 \\
\hline & $(2.2756)$ & $110.27)$ & $(155.80)$ & $(50.66)$ & $(180.00)$ & $(180.00)$ & $(180.00)$ \\
\hline \multirow[t]{2}{*}{8} & 3.1 & .66 & 70.68 & 70.68 & -63.69 & 180.00 & 67.57 \\
\hline & $(3.1140)$ & $(92.24)$ & $(70.84)$ & $(7$ & $(-64.02)$ & $(180.00)$ & (67.93) \\
\hline \multirow[t]{2}{*}{9} & 2.5228 & 112.02 & 127.69 & 127.69 & 0.00 & -90.00 & 90.00 \\
\hline & $(2.5154)$ & 112.03 & $(127.79)$ & $(127.79)$ & $(0.00)$ & $(-90.00)$ & $(90.00)$ \\
\hline \multirow[t]{2}{*}{10} & 2.6846 & 112.87 & 127.86 & 127.86 & 0.00 & 0.0 & 180.0 \\
\hline & $(2.6830)$ & $(112.92)$ & $(127.95)$ & $(127.95)$ & $(0.00)$ & $(0.00)$ & $(180.00)$ \\
\hline 11 & 3.3845 & 114.33 & 52.66 & 52.66 & 0.00 & -90.00 & -90.00 \\
\hline
\end{tabular}


TABLE III: Comparison of selected geometrical parameters. Bond lengths are in $\AA$, angles in degrees. The first row corresponds to results from reference 13 (MP2/IOM, counterpoise corrected), the second to reference 15 (MP2/6-311+G(d,p) for structures 1, 4 and 9 and MP2/6-31+G(d,p) else), the third corresponds to this work and the last one to reference 14 $(\operatorname{CCSD}(\mathrm{T}) / \mathrm{TZ2P}(f, d)+$ dif $)$.

\begin{tabular}{|c|c|c|c|c|c|}
\hline method & structure & $r_{\mathrm{O}_{1} \mathrm{O}_{2}}$ & $r_{\mathrm{H}_{2} \mathrm{O}_{1}}$ & $r_{\mathrm{H}_{2} \mathrm{O}_{2}}$ & $\theta_{\mathrm{O}_{1} \mathrm{H}_{2} \mathrm{O}_{2}}$ \\
\hline MP2 & 1 & 2.896 & 0.965 & 1.944 & 176.7 \\
\hline MP2(IOM) & 1 & 2.936 & 0.9572 & 1.984 & 172.4 \\
\hline MP2-R12 & 1 & 2.907 & 0.9661 & 1.947 & 171.68 \\
\hline $\operatorname{CCSD}(\mathrm{T})$ & 1 & 2.909 & 0.9653 & 1.948 & 172.92 \\
\hline MP2 & 3 & 2.916 & 0.968 & 1.970 & 169.1 \\
\hline MP2(IOM) & 3 & 2.949 & 0.9572 & 2.011 & 166.2 \\
\hline MP2-R12 & 3 & 2.916 & 0.9647 & 1.971 & 165.70 \\
\hline $\operatorname{CCSD}(\mathrm{T})$ & 3 & 2.930 & 0.9640 & 1.981 & 167.59 \\
\hline MP2 & 4 & 2.770 & 0.962 & 2.278 & 112.0 \\
\hline MP2(IOM) & 4 & 2.838 & 0.9572 & 2.307 & 114.3 \\
\hline MP2-R12 & 4 & 2.807 & 0.9619 & 2.270 & 114.35 \\
\hline $\operatorname{CCSD}(\mathrm{T})$ & 4 & 2.822 & 0.9616 & 2.280 & 114.84 \\
\hline MP2 & 5 & 2.746 & 0.966 & 2.275 & 109.6 \\
\hline MP2(IOM) & 5 & 2.810 & 0.9572 & 2.310 & 111.8 \\
\hline MP2-R12 & 5 & 2.777 & 0.9616 & 2.273 & 111.79 \\
\hline $\operatorname{CCSD}(\mathrm{T})$ & 5 & 2.794 & 0.9614 & 2.281 & 112.52 \\
\hline MP2 & 6 & 2.735 & 0.965 & 2.276 & 108.5 \\
\hline MP2(IOM) & 6 & 2.786 & 0.9572 & 2.311 & 109.9 \\
\hline MP2-R12 & 6 & 2.755 & 0.9613 & 2.275 & 109.98 \\
\hline $\operatorname{CCSD}(\mathrm{T})$ & 6 & 2.760 & 0.9611 & 2.276 & 110.27 \\
\hline MP2 & 8 & 3.382 & 0.963 & 3.144 & 90.9 \\
\hline MP2(IOM) & 8 & 3.392 & 0.9572 & 3.213 & - \\
\hline MP2-R12 & 8 & 3.344 & 0.9592 & 3.159 & 92.66 \\
\hline $\operatorname{CCSD}(\mathrm{T})$ & 8 & 3.294 & 0.9594 & 3.114 & 92.24 \\
\hline MP2 & 9 & 2.918 & 0.960 & 2.462 & 112.03 \\
\hline MP2(IOM) & 9 & 3.064 & 0.9572 & 2.591 & - \\
\hline MP2-R12 & 9 & 3.017 & 0.9595 & 2.523 & 112.02 \\
\hline $\operatorname{CCSD}(\mathrm{T})$ & 9 & 3.010 & 0.9596 & 2.515 & 112.03 \\
\hline MP2 & 10 & 3.105 & 0.963 & 2.630 & 112.97 \\
\hline MP2(IOM) & 10 & 3.242 & 0.9572 & 2.762 & - \\
\hline MP2-R12 & 10 & 3.182 & 0.9590 & 2.685 & 112.87 \\
\hline $\operatorname{CCSD}(\mathrm{T})$ & 10 & 3.182 & 0.9591 & 2.683 & 112.92 \\
\hline
\end{tabular}




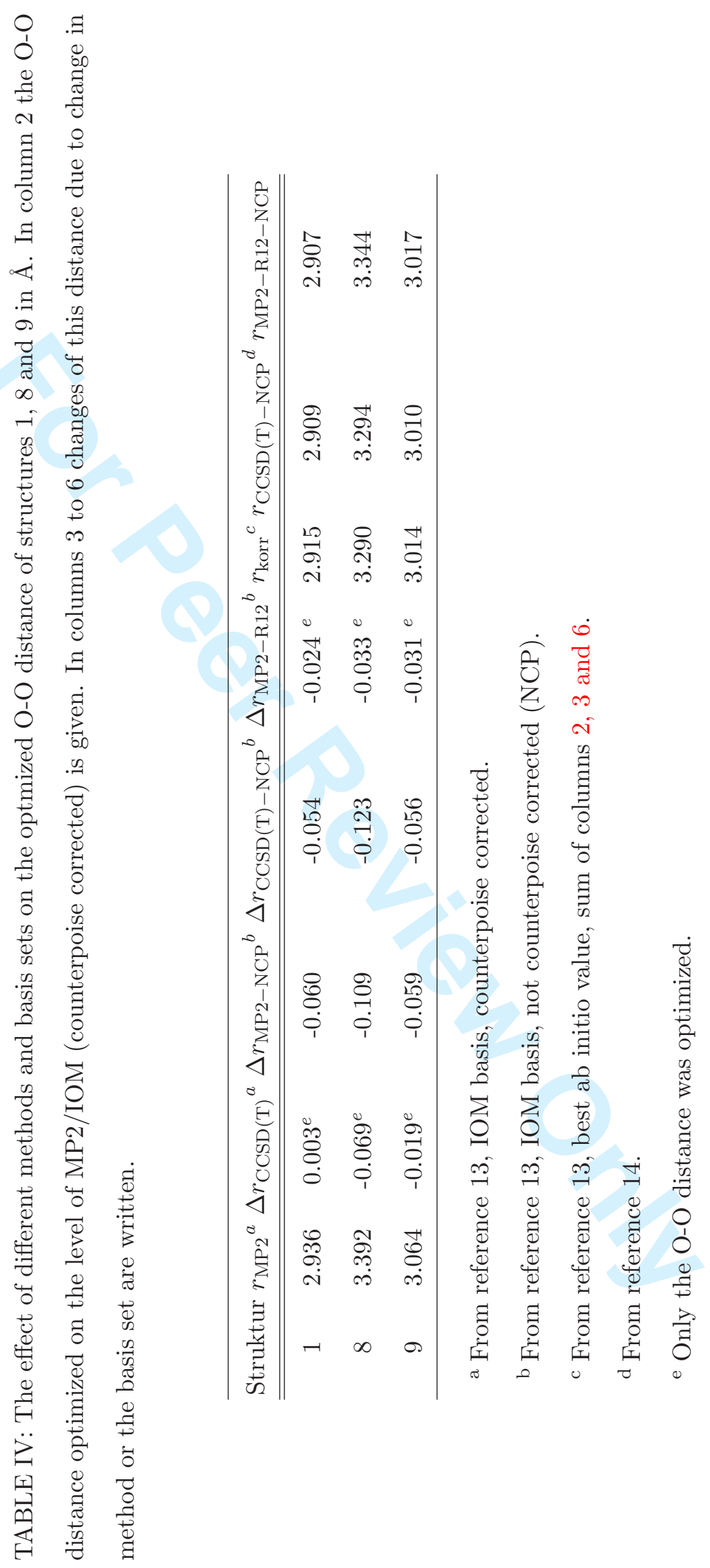




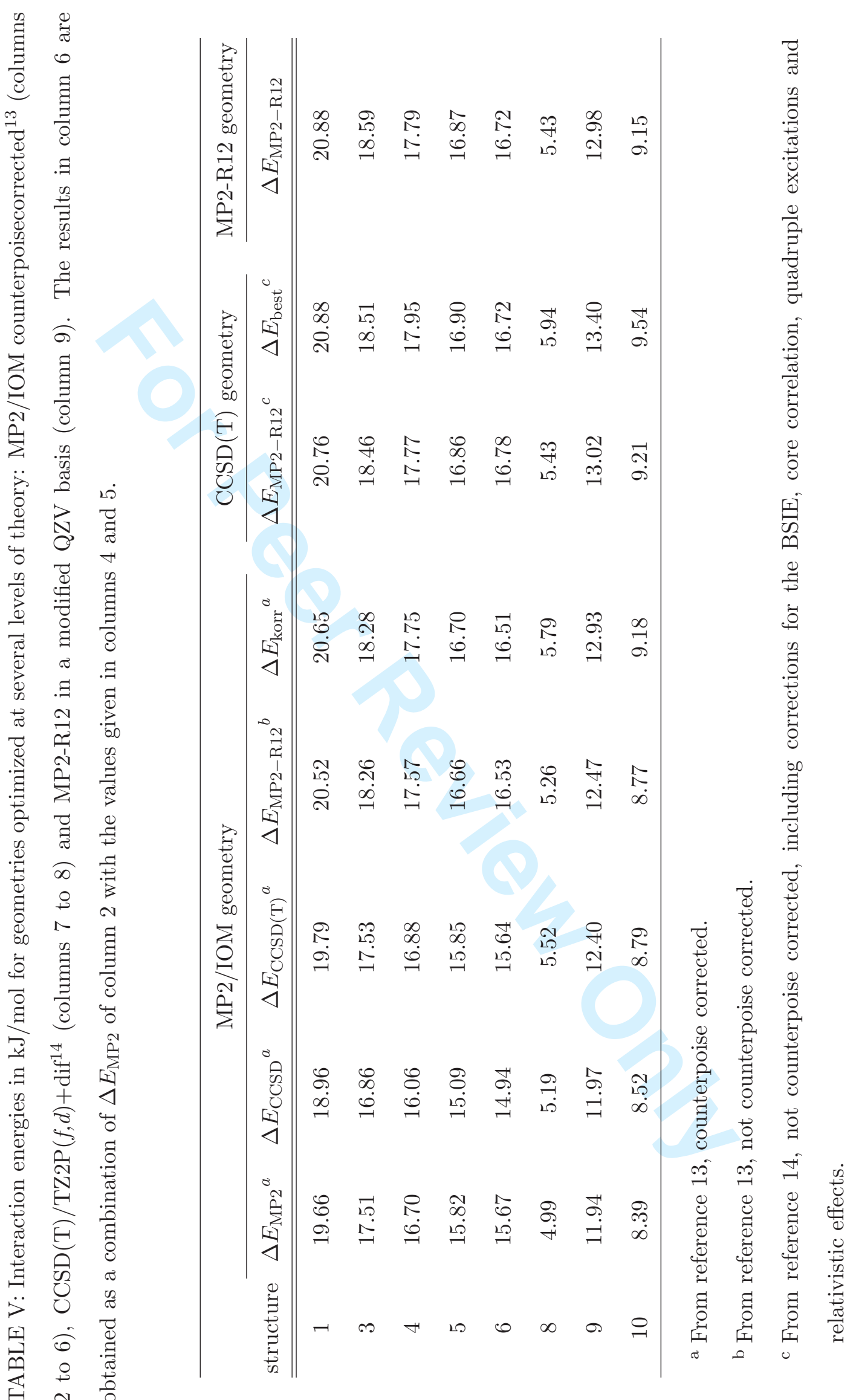


